\title{
Simulation research on dynamic performance of the new type high-pressure solenoid valve
}

\author{
Qihui YU ${ }^{1,2^{*}}$, Qiancheng WANG ${ }^{1}$, Kaifei ZHANG ${ }^{3}$, Weiwei ZHENG $^{4}$
}

1 School of Mechanical Engineering, Inner Mongolia University of Science and Technology, baotou, China

2 The State Key Laboratory of Fluid Power and Mechatronic Systems, Hangzhou, China

3 College of Mechanical and Electrical Engineering, Henan Agricultural University, 450002, Zhengzhou, China

4 College of Mechanical and Electrical Engineering, Henan radio and television University, Zhengzhou, China,

*Corresponding Author: Qihui YU, Baotou, 014010,China; yqhhxq@163.com

\begin{abstract}
:
To improve energy density, the transportation, storage, and operations of hydrogen, methane, and compressed air vehicles currently require high-pressure compression. High-pressure solenoid valve becomes the vital element to above system. In order to reduce leakage and aerodynamic force influence, a new type high-pressure solenoid valve was proposed. The simulation model which included electromagnetic model, aerodynamic force model was established by means of the nonlinear mathematic models. Using the software MATLAB/Simulink for simulation, the dynamic response characteristics of high-pressure pneumatic solenoid valve were obtained under different pulse width modulation (PWM) input control signals. Results show that, first of all, the new type of high-pressure solenoid valve can meet the switch requirement. Secondly, the opening movement and closing movement of the spool lags the PWM rising signal, and the coil current fluctuates significantly during the movement of the spool. Lastly, on/off status of high-pressure valve cannot be represented by the duty cycle. This research can be referred in the design of the high-pressure solenoid valve..
\end{abstract}

Keywords: high-pressure solenoid valve; dynamic response performance; pulse width modulation; the duty cycle

\section{Introduction}

To improve energy density, the transportation, storage, and operations of hydrogen, methane, and compressed air vehicles currently require high-pressure compression. The use of electronic control technology is a major direction for the development of above systems. To gas distribution system, the high-pressure solenoid valve is the vital part. The control performance of the gas distribution system is mainly determined by the applied high-pressure solenoid valve. Its dynamic response characteristics directly affect the efficiency and reliability of the system.

Normally, the electromagnetic switch valve is used as the pilot valve of the control stage ${ }^{[1]}$, which is directly controlled by the pulse width modulation (PWM) method and the output pulse flow rate is the control variable of the power level pilot valve. Most researches are based on simulation under the no-load condition of the solenoid valve ${ }^{[2-3]}$, and the pressure characteristics of the fluid is neglected. In order to reduce leakage and aerodynamic force influence, a new type high-pressure solenoid valve was proposed. The simulation model which included electromagnetic model, aerodynamic force model was established by means of the nonlinear mathematic models. Using the software MATLAB/Simulink for simulation, the dynamic response characteristics of high-pressure pneumatic solenoid valve were obtained under different pulse width modulation (PWM) input control signals. This research can be referred in the design of the high-pressure solenoid valve.

\section{Working principle of high-pressure solenoid valve}

To reduce leakage and aerodynamic force influence, the main structure consists two parts: one is pressure compensated which is adopted slide valve mechanism; the other is seal which is adopted poppet valve mechanism which has good sealing performance. Figure 1 is the structural schematic diagram of the force balanced high pressure solenoid valve which was used in high pressure pneumatic engine. 


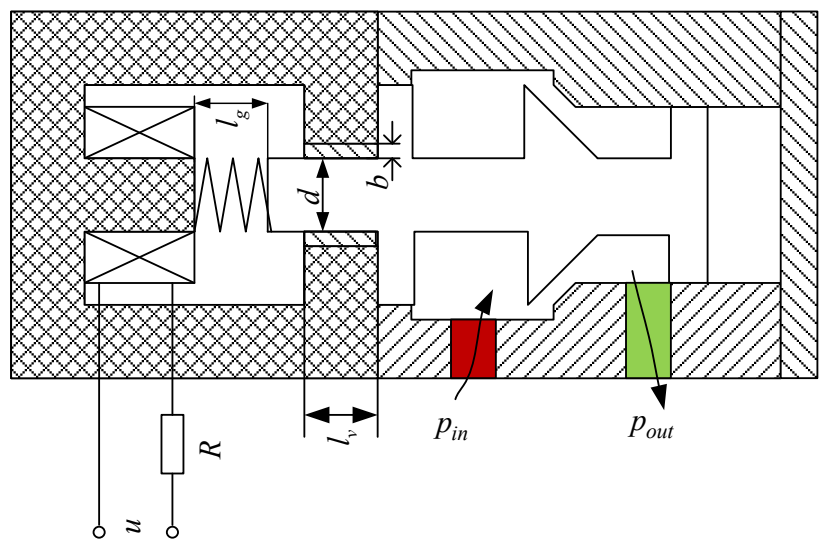

Figure 1 The structure of high-pressure solenoid valve

The valve working process can be divided into the following two stages:

\subsection{Opening phase}

According to the requirements of gas distribution control, the electronic control unit sends control pulses to the drive module of the solenoid valve at a specific time. The drive module provides a high peak drive voltage, and the solenoid valve generates an electromagnetic force on the spool. When the electromagnetic force is greater than the preload of the return spring, the spool moves upward to quickly open the valve.

\subsection{Closing phase}

When the control pulse is terminated, the driving voltage is cut off, and the solenoid valve is de-energized, the electromagnetic force quickly disappears. Under the action of the return spring, the spool moves downward until the solenoid valve is completely closed.

The high-pressure solenoid valve used in the pneumatic engine gas distribution system should have good fast response performance, the main reasons are the following aspects:

(1) The rapid opening of the solenoid valve is conducive to ensuring the accurate timing of the valve and the rapid formation of high pressure; the quick closing of valve is helpful to ensure the quick cutting off of highpressure gas. The slow opening of the valve makes the highpressure gas entering the pneumatic engine unable to be distributed according to the predetermined control law, thus greatly reducing the economy and efficiency of the pneumatic engine.

(2) The response time of the closing process and opening process of the solenoid valve has a great influence on the air distribution of the pneumatic engine. Especially when the air supply pressure is high and the engine's angular speed is very fast, in order to obtain stable output power, the response time of the solenoid valve should be appropriately short.

From the above analysis, it can be seen that shortening the response time of the closing and opening process of the solenoid valve is the key to achieving good performance of the electronically controlled gas distribution system, and also the key to improving the comprehensive performance of the pneumatic engine.

\section{Theoretical analysis of driving characteristics of high-pressure solenoid valve}

The flow of gas in a valve is a thermodynamic process of a complex open system. To simplify the model, the following assumptions were made ${ }^{[4]}$ :

(1) Pressure fields and velocity fields in chambers are homogenous;

(2) Leakage and pressure loss are ignored;

(3) The working medium is an ideal air that followed the equation of an ideal air state;

(4) Impact contact influence is ignored.

\subsection{Electromagnetic characteristic equation of solenoid} valve

The working process of the solenoid valve is simplified into the electromagnetic circuit equation and the equation of motion ${ }^{[5-11]}$.

In general, the inductance of the electromagnet can be expressed by equation (1):

$$
L=\mu_{0} \pi D^{2} N^{2} l_{v} / 4 l_{v}\left(l_{0}-x\right)+r D
$$

where $\mu_{0}$ is the vacuum permeability, $D$ is armature diameter, $N$ is coil turns, $l_{v}$ is the length of valve spool armature, $r$ is the average width of the working air gap, $l_{0}$ is the maximum length of the working air gap, $x$ is spool displacement.

The derivative of equation (1) is obtained:

$$
\frac{d L}{d x}=4 \mu_{0} \pi D^{2} N^{2} l_{v}^{2} /\left[4 l_{v}\left(l_{0}-x\right)+r D\right]^{2}
$$

Using the principle of virtual displacement and setting the amount of change in the air gap of the electromagnet, the mechanical work done by electromagnetic force can be obtained. The mechanical work is equal to the change of the total magnetic energy of the system. According to equation (3), the electromagnetic force can be obtained as shown in equation (4):

$$
\begin{aligned}
& F d x=d W=1 / 2 i^{2} d L \\
& F=\frac{1}{2} i^{2} d L / d x
\end{aligned}
$$

where $d x$ is the amount of change in the air gap of the electromagnet, $F d x$ is mechanical work, $d W$ is the change in total magnetic energy, $i$ is the current in the solenoid coil.

The current in the solenoid valve coil can be obtained by equation (5):

$V_{s}=i R+L \frac{d i}{d t}+i \frac{d L}{d t}$

where $V_{s}$ is the coil excitation voltage, $R$ is resistance of the coil, $t$ is time.

Before the armature is moved, $d L / d t=0$; once the armature is attracted, the kinematic counter electromotive force will be generated. At this time, the spool motion 
voltage equation is as follows:

$e=i \frac{d L}{d t}=v i \frac{d L}{d x}$

where $v$ is the movement speed of the spool.

\subsection{Dynamic equation of solenoid valve}

The equation of motion of the solenoid valve can be expressed as follows:

$F_{\text {mag }}-k_{s} x_{0}-k_{s} x-b_{1} \dot{x}-F_{f l u}=m a$

where $a$ is motion acceleration, $F_{\text {mag }}$ is electromagnetic force of the solenoid valve, $k_{s}$ is stiffness of the return spring, $x_{0}$ is initial pretension length of the return spring, $b_{1}$ is the viscosity coefficient of air, $F_{f l u}$ is the aerodynamic force suffered by the spool, $m$ is spool mass.

As can be seen from equation (7), in the opening process of the solenoid valve, in order to shorten the response time, it is expected that the electromagnetic force will increase, and the air force on the valve core, the return spring force, and the mass of the valve core will decrease. In the closing process, in order to shorten the response time, it is hoped that the solenoid valve acting force will quickly reduce to zero, the return spring acting force will increase, the aerodynamic force of the valve core will increase, the gas resistance will decrease, and the quality of the valve core will decrease.

\subsection{Aerodynamic equation of solenoid valve spool}

From the spool structure shown in Figure 1, the principle diagram of the mechanism can be obtained as shown in Figure 2:

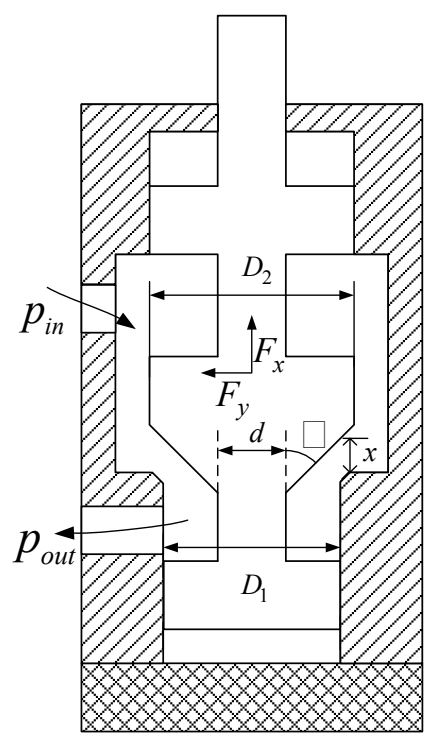

Figure 2 The schematic structure of the poppet valve

The aerodynamic force received by the spool can be derived from Euler's law of momentum, as shown in Equation 8 below:

$F_{f l u}=-\frac{d}{d t}\left[\int_{V} \rho \vec{u} d V\right]-\int_{A_{e}} \vec{u}\left(\rho \vec{u} d A_{e}\right)$ where $V$ is volume of the control body formed by the inner cavity of the valve core, $\rho$ is gas density, $\vec{u}$ is gas flow rate, $A_{e}$ is equivalent cross-sectional area of the flow crosssection.

The above formula is mainly composed of two parts, among which the first term on the right represents the transient aerodynamic force required for the valve core inner chamber to control the gas in the body to accelerate (or decelerate), the second term represents the steadystate aerodynamic force caused by the different velocities of the gas at different positions. Because the transient aerodynamic force of the spool is mainly caused by the speed change of the spool, and the air gap of the spool of the switching valve is very short, the maximum speed that the spool can reach is limited, so the influence of the transient aerodynamic force is ignored here.

Finally, the aerodynamic force experienced by the spool can be expressed as:

$F_{f l u}=\int_{D_{1} / 2}^{D_{2}} 2 p \pi r \cos \alpha \sin \alpha d r-\bar{\rho} q_{m g} v_{1} \cos \alpha$

$p=p_{\text {in }}-\frac{6 \eta q_{m g}}{\bar{\rho} \pi x^{3} \sin ^{4} \alpha} \ln \frac{2 r}{D_{1}}$

$q_{m g}=\frac{\bar{\rho} \pi x^{3} \sin ^{4} \alpha\left(p_{\text {in }}-p_{\text {out }}\right)}{6 \eta \ln \left(D_{1} / D_{2}\right)}$

$v_{1}=c_{v} \sqrt{\frac{2}{\bar{\rho}}\left(p_{\text {in }}-p_{\text {out }}\right)}$

$\bar{\rho}=\left(p_{\text {in }}+p_{\text {out }}\right) / 2 R_{g} T$

where $p_{\text {in }}$ is pressure of the intake, $p_{\text {out }}$ is pressure of the exhaust, $x$ is distance the spool moves, $\alpha$ is half the cone angle of the cone valve, $\eta$ is dynamic viscosity of a gas, $R_{\mathrm{g}}$ is gas constant, $T$ is temperature of the environment, $c_{v}$ is speed factor, $p$ is velocity distribution at the orifice, $q_{m g}$ is the mass flow of gas through the orifice, $\bar{\rho}$ is average density of the gas in the valve. The remaining structural parameters can be seen in Figure 2 .

Considering the continuity characteristics of aerodynamic force, assuming that the steady-state aerodynamic force maintains a linear relationship with its opening within the spool action range, so a correction coefficient is introduced in this way, and the above aerodynamic force is corrected as:

$F_{p}=\left(k_{f 1}+k_{f 2} x / x_{m}\right) F_{f l u}$

Consider the boundary conditions when the correction coefficient is satisfied: when the opening and closing of the valve is $x \rightarrow 0, F_{P}=k_{f 1} F_{f}=\mathrm{F}_{\mathrm{f}}$, so take $k_{f 1}=1$; When the opening degree of the on-off valve is the maximum value, $F_{P}=$ $\left(1+k_{f 2}\right) F_{f}$, in this paper, the empirical value $k_{f 2}=1.15$ is used. In this way, the aerodynamic force of the solenoid valve can be obtained.

From the above analysis, the block diagram of the nonlinear model of the spool can be obtained as follows: 


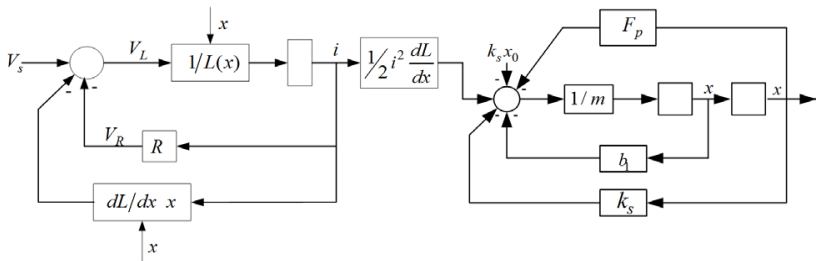

Figure 3 Non-linear model of the solenoid valve spool

\section{Dynamic characteristics simulation analysis}

The high-pressure solenoid valve performs "on" and "off" actions according to the PWM control electrical signal. It can be seen that the flow rate of the on-off valve is proportional to the duty cycle $(\tau=$ pulse width / pulse period) of the input voltage signal. The larger the duty cycle, the greater the corresponding control flow rate. Therefore, to study the dynamic performance of the electromagnetic switch valve, it is necessary to simulate the movement of the spool under the PWM control signals of different duty cycles. In this study, the step signal is used to excite the PWM signal module. The PWM signal controls the on and off of the electromagnetic switch valve.

In this study, the simulation parameters were set to $3 \mathrm{MPa}$ gas pressure, PWM signal frequency $20 \mathrm{~Hz}$, and PWM signal duty cycle $\tau$ were $0.25,0.5,0.75$, respectively. The relationship between the PWM signal of the switching valve, the coil current $i$ of the electromagnet, and the displacement $S$ of the valve core is shown in Figures 4-6.
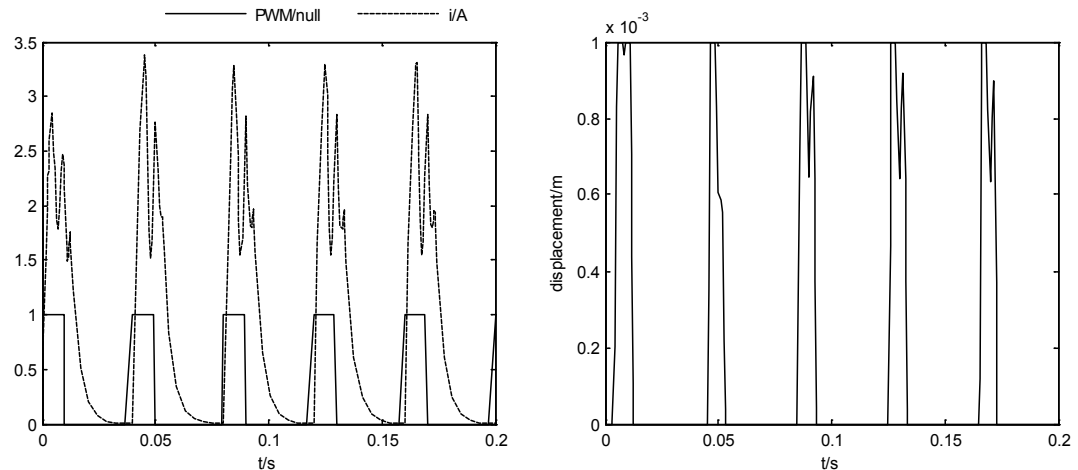

Figure 4 The solenoid valve PWM signal, coil current and spool displacement curve ( $\tau=0.25)$
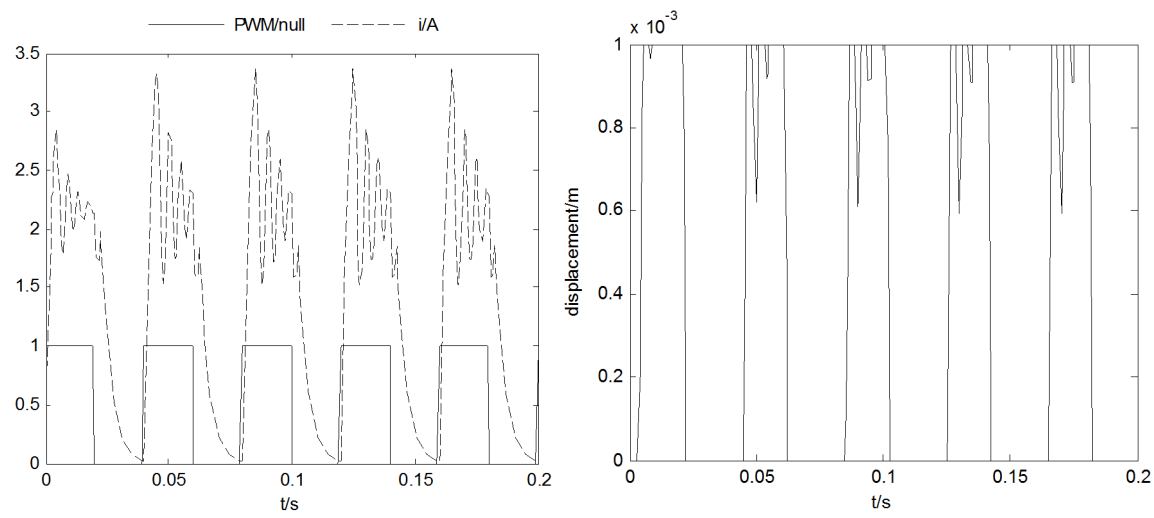

Figure 5 The solenoid valve PWM signal, coil current and spool displacement curve ( $\tau=0.5)$
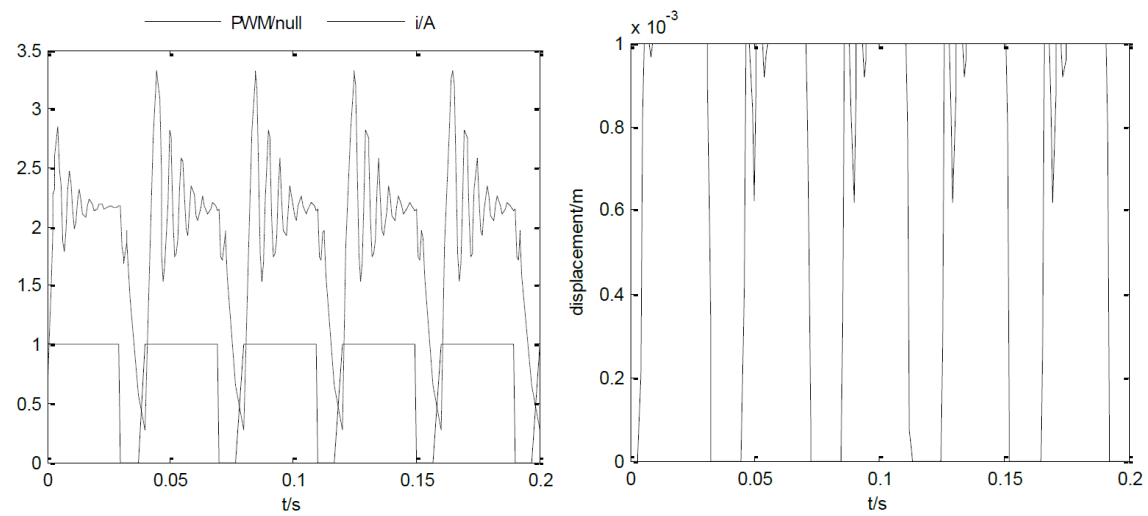

Figure 6 The solenoid valve PWM signal, coil current and spool displacement curve ( $\tau=0.75$ ) 
By analyzing the relationship between the PWM signal with different duty cycles, coil current, and spool displacement in Figure 4-6, the following conclusions can be drawn:

(1) The spool opening movement lags the PWM rising signal, and the coil current fluctuates significantly during the movement of the spool. The reason is that when PWM input high level, the coil is connected to the power supply voltage, where the current gradually increases from zero, and the law of magnetic flux growth is the same as the current. When the current increases so that the electromagnetic attraction generated is sufficient to move the armature, the armature begins to move. After the armature moves, the air gap decreases, the magnetic resistance in the air gap changes, and the coil inductance changes, causing a small back electromotive force, which reduces the coil current. This caused the coil current to fluctuate.

(2) The spool closing movement also lags the PWM falling signal, and the coil current fluctuates significantly during the spool movement. The reason is when PWM input low level, the excitation in the coil is lost, and the magnetic flux begins to decay. Due to the induced potential and the eddy current in the iron core, the electromagnetic attraction is gradually reduced. When the suction force is not enough to hold the armature, the armature starts to release, the air gap increases, the magnetic resistance in the air gap changes, and the coil inductance changes, causing a small positive potential to increase the coil current. This causes the coil current to fluctuate when the spool is closed.

(3) When other parameters are unchanged, the relationship between the duty cycle of the PWM signal, the coil current and the displacement of the spool has the following rules: with the decrease of $\tau$, the duration of the high level of the PWM input signal shortens, and the coil the maximum value that the current can reach is decreasing. When $\tau$ is reduced to a certain value, the force of the electromagnet is not enough to move the armature then the spool cannot be opened. At this time, the solenoid valve will be in a normally closed state, and the PWM signal cannot be play a controlling role; with the increase of $\tau$, the high level duration of PWM input signal is extended. When the low level of PWM signal is not enough to close the valve core, the coil current will start to increase. The valve core cannot be closed, and the PWM signal cannot play a control role at this time.

\section{Conclusion}

Through the above research on the dynamic characteristics of the solenoid valve and the simulation results, the following conclusions can be drawn:

(1) Since the presence of the high-speed switching valve induced current causes the opening and closing of the spool to lag behind the PWM control signal, the duty ratio $\tau$ cannot be completely changed arbitrarily.

(2) Due to the time delay, the duty cycle cannot fully represent the switching state of the spool.

In summary, the combination of MATLAB / Simulink can better complete the simulation analysis of the modeling process of pneumatic high-pressure high-frequency solenoid valves. Provide a basis for the control efficiency and control accuracy of high-pressure high-frequency pneumatic solenoid valves.

Author Contributions: Qihui YU contributed to the conception of the study; Qiancheng WANG contributed significantly to analysis and manuscript preparation; Kaifei ZHANG performed the simulation model; and Yiwei Zheng helped perform the analysis with constructive discussions. Conflict of Interest: There is no conflict of interest regarding the publication of this paper.

Acknowledgments: The research work presented in this paper is financially supported by a grant (NJZZ18139) from the scientific research project of Universities in Inner Mongolia, a grant (2018BS05003) from the Natural Science Foundation of Inner Mongolia, and a grant (2017QDL-B07) from Inner Mongolia University of Science and Technology Innovation Fund Project.

\section{References}

[1] Lihuan, G; Kaizhang, S; Gang, Z. The new high speed digital switch valve is a multi - way reversing valve. Journal of Lanzhou University of Technology. 2006, 32(3), 56-58, DOI: CNKI:SUN:GSGY.0.2006-03-015

[2] Yugui, L;Xiaoming, Y;Xuejie, G. Study on static characteristics of PWM high speed switching valve. Journal of taiyuan heavy machinery college. 2002, 23(1), 68-71,DOI: 10.3969/j.issn.1673-2057.2002.01.017

[3] Zhong, L;Weiping, L; Qian, H. Modeling and simulation of dynamic response of high speed switching solenoid valve. Journal of Natural Science of Hunan Normal University. 2009, 32(3), 53-57, DOI: 10.3969/j.issn.10002537.2009.03.013

[4] Gang Y; Youbin S; Baoren L. Numerical simulation on dynamic characteristics of Extra-high pressure pneumatic Blowing valve. China Mechanical Engineering. 2012, 23(1): 42-45 (In Chinese) DOI: 10.3969/j.issn.1004 132X.2012.01.009.

[5] Lihui, W; Zhixin, Y; Minghui, Y. Simulation and testing of dynamic characteristics of digital closed loop fiber optic current transformer. Chinese Journal of Scientific Instrument. 2010, 31(8), 1890-1895, DOI: CNKI:SUN:YQXB.0.2010-08-033

[6] Yi, L; Bin, G; Xiaowang, P. Study on the comprehensive performance test system of direct-acting pneumatic solenoid valve. Chinese Journal of Scientific Instrument. 2011, 32(4), 795-800, DOI: CNKI:SUN:YQXB.0.2011-04-013

[7] Xiaowang, P; Min, L; Bin, G. Simulation and experimental study of dynamic characteristics of pneumatic solenoid valve. Journal of China University of Metrology. 2010, 21(3), 232-236, DOI: CNKI:SUN:ZGJL.0.2010-03-010

[8] Xiao L; Wenjie, N. Design calculation of solenoid valve. Electric Switch. 2008, (3), 11-13, DOI: CNKI:SUN:DQKG.0.2008-03-006 
[9] Farong, D; Juan, H. Simulation and Response Analysis of High-speed Solenoid Valve for EUI. Science Technology and Engineering. 2011, 11(5), 1080-1083, DOI: CNKI:SUN:KXJS.0.2011-05-038.

[10] Ting, C. Simulation of high pressure common rail solenoid valve of diesel engine and its driving circuit. Beijing
Jiaotong University. 2009, DOI: 10.7666/d.y1577655

[11] Zhihong, B; Yuhu, Z. Simulation and analysis of dynamic characteristics of electromagnet. Journal of Electric Power.2009, 19(3), 200-201, DOI: 10.3969/j.issn.10056548.2004.03.006 